\title{
INVESTIGACIÓN FORMATIVA PARA ESTUDIANTES DE INGENIERÍA UTILIZANDO ROBÓTICA
}

\section{FORMATIVE RESEARCH FOR ENGINEERING STUDENTS BY USING ROBOTICS}

\author{
PhD. Liliana Fernández Samacá, MSc. Luis Ariel Mesa Mesa \\ PhD. Wilson Javier Pérez Holguín. \\ Universidad Pedagógica y Tecnológica de Colombia - UPTC \\ Grupo de Investigación en Robótica y Automatización Industrial GIRA. \\ Calle 4 Sur No. 15-134. Sogamoso, Boyacá, Colombia. \\ Tel.:(+57 8) 770 5450, (+57 8) 7706896 , Ext. 2621. \\ E-mail: \{ariel.mesa, liliana.fernandez, wilson.perez\{@uptc.edu.co
}

\begin{abstract}
Resumen: Este artículo describe una experiencia centrada en la formación en investigación para estudiantes de primeros semestres de Ingeniería Electrónica a través de la Robótica. El documento presenta una descripción de los entornos educativos desarrollados para fomentar el aprendizaje de los conceptos relacionados con la robótica y la investigación científica. Los resultados muestran mejoras significativas en el rendimiento académico y de investigación de los estudiantes involucrados, especialmente en la mejora de sus habilidades y conocimientos, así como en la motivación hacia el proceso de aprendizaje.
\end{abstract}

Palabras clave: Investigación Formativa, Educación en Ingeniería, Robótica.

Abstract: This paper describes an experience focused on research training for freshman and junior Electronic Engineering students. This document introduces a brief description of the educational environments developed to encourage the learning of concepts related to robotics and scientific research. The results show significant improvements in academic and research performance of involved students, especially in the enhancement of their skills and knowledge, as well as the motivation towards the learning process.

Keywords: Research Training, Engineering Education, Robotics.

\section{INTRODUCCIÓN}

El diseño de enfoques educativos y de herramientas que motiven a los estudiantes a desarrollar habilidades para la investigación, la innovación y la creatividad, es uno de los retos que tiene actualmente la Educación en Ingeniería. Una de las formas de enfrentar este desafío, es el desarrollo de ambientes de aprendizaje que incentiven la formación de una actitud investigativa desde un ejercicio real que involucre a los estudiantes en un proceso de investigación.
La formación en investigación se vuelve más exigente en los primeros semestres de las ingenierías, dado que en la mayoría de los casos en estos semestres se tienen otros retos como la inducción del estudiante a la educación superior, que en muchos casos supone un cambio en su entorno social o familiar y de sus prácticas de enseñanza y aprendizaje. Es así como los programas de ingeniería, además del aprendizaje disciplinar, deben ocuparse de proveer ambientes de aprendizaje que faciliten la adaptación de los estudiantes a la vida universitaria (Restrepo, 2001). 
Esto se hace especialmente necesario en áreas con mayores niveles de exigencia, tales como las matemáticas, la física, los circuitos o la computación, entre otras.

Por otra parte, para la mayoría de los estudiantes de primeros semestres de los programas de ingeniería, el concepto de investigación puede resultar abstracto, e incluso atemorizante, ya que muchas veces se desconoce lo que esta tarea implica dentro de la formación. Por esta razón, para muchos estudiantes considerar el aprendizaje a través de la investigación, es un desafío mayor.

En este artículo se presenta un ambiente de aprendizaje formativo en investigación que toma la robótica como eje dinamizador del aprendizaje de los estudiantes en un programa de Ingeniería Electrónica. El ambiente define desafíos de investigación en los cuales estudiantes de primeros semestres se involucran en diferentes actividades de formación y aplicación de la robótica. Se ha mostrado en diversos trabajos como los presentados por (Ruiz, 2007) y (Barrera y Mesa, 2013), que la robótica puede resultar atrayente y motivante para la mayoría de estudiantes de programas relacionados con el desarrollo de tecnología. Sin embargo, a pesar de este estímulo, no siempre resulta sencillo en el entorno universitario enseñar y comprender varios de los aspectos que se manejan en esta área, considerando, por ejemplo, que en los primeros semestres los estudiantes no han alcanzado un alto nivel de fundamentación en cálculo, circuitos eléctricos, electrónica, máquinas eléctricas e instrumentación.

Los resultados presentados se basan en la experiencia del Grupo de Investigación en Robótica y Automatización Industrial GIRA Uptc, con estudiantes de primeros semestres. El grupo GIRA se ha caracterizado por ser uno de los grupos de investigación de la Universidad Pedagógica y Tecnológica de Colombia, Sogamoso, que cuenta con un importante número de estudiantes vinculados en su semillero de investigación (S_GIRA) y se destaca por participar continuamente en diversos eventos de semilleros a nivel regional, nacional e internacional.

\section{DESCRIPCIÓN DE LOS AMBIENTES DE FORMACIÓN EN INVESTIGACIÓN}

En los últimos años los robots han sido utilizados no solamente para la automatización de procesos industriales, militares o de investigación, sino que también han sido adoptados ampliamente como una herramienta didáctica dentro de las aulas de clase, especialmente en los programas de ingeniería y tecnología.

El uso de la robótica en este contexto, implica múltiples ayudas al docente que involucran el abordaje de componentes teóricos y prácticos que motivan y facilitan la asimilación de conceptos. Sin embargo, la robótica y sus conceptos asociados pueden no ser fáciles de entender si se tiene en cuenta la gran cantidad de disciplinas que convergen en ella (electrónica, mecánica, sistemas y computación, control automático, TIC's, inteligencia de artificial, etc.).

Los semilleros de investigación en los programas de pregrado en ingeniería son espacios dedicados a propiciar que estudiantes, especialmente de primeros semestres, se vinculen y aprendan, de manera lúdica, conceptos relacionados con el área de desempeño (circuitos, programación, máquinas eléctricas, control automático, electrónica. etc.), además de desarrollar habilidades y capacidades para la investigación (Restrepo, 2001).

La formación en investigación de los estudiantes de primeros semestres es uno de los objetivos más importantes dentro del trabajo con semilleros de investigación, ya que contribuye a que los grupos de investigación y los programas de pregrado cuenten con el personal que permita plantear y ejecutar investigaciones de alto nivel, mediante el apoyo al trabajo de los investigadores de mayor experiencia y nivel de formación.

Adicionalmente, la formación temprana en investigación, permite aprovechar por más tiempo las habilidades y capacidades de los estudiantes, focalizándolas de acuerdo con los intereses del grupo de investigación y de la Universidad.

Por otra parte, es importante considerar que los estudiantes que ingresan cada semestre al semillero, poseen niveles de conocimiento y habilidades disimiles, lo que hace que la labor formativa sea más exigente. Asimismo, puede ocurrir que se vinculen personas que, a pesar de estar en primeros semestres de pregrado, cuentan con amplios conocimientos técnicos y/o tecnológicos, que pueden contribuir significativamente al desarrollo de diversas actividades. Es claro entonces, que es necesario abordar el reto de mantener a todos los nuevos integrantes motivados, y canalizar de la mejor 
forma sus destrezas y conocimientos para beneficio del grupo de investigación.

La Figura 1 muestra algunos de los actores que generalmente intervienen en el ambiente universitario de aprendizaje de un estudiante de primeros semestres, que se vincula con el semillero de investigación. La relación con cada una de estas personas contribuye a la asimilación de conceptos y al afianzamiento de su formación en investigación.

No se incluyen en el diagrama las relaciones con otros actores que pueden aparecer fruto de la ejecución de otras actividades que usualmente se desarrollan en un grupo de investigación. El grupo GIRA por ejemplo, desarrolla continuamente proyectos y trabajos relacionados con la robótica educativa, creando interacciones con profesionales de diversas disciplinas. En este diagrama se omiten también las contribuciones que el estudiante que participa como semillero de investigación pueda aportar al resto de los actores, ya que el interés de este trabajo se focaliza solo en su formación dentro del grupo.

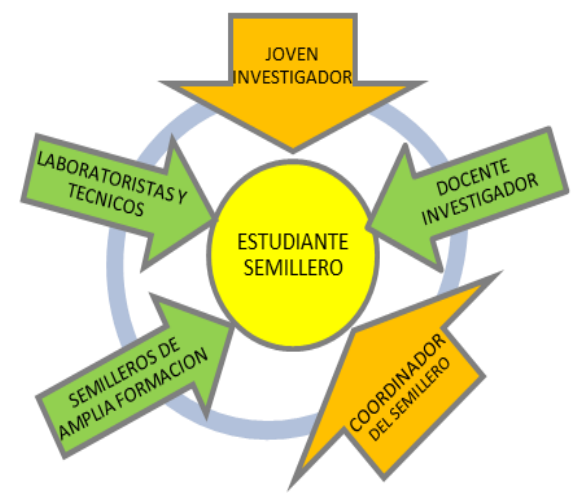

Fig. 1. Actores intervinientes en el proceso de formación de un estudiante semillero en el ambiente universitario.

En estos ambientes se emplean múltiples estrategias y herramientas didácticas para lograr que los nuevos estudiantes se relacionen adecuadamente con el mundo de la robótica.

Frecuentemente se emplean concepciones constructivistas como el aprendizaje basado en problemas (Problem-Based Learning), el aprendizaje basado en proyectos (Project-Based Learning) y el aprendizaje activo, entre otras, siendo normal que se presenten mezclas de éstas, con el objetivo de mejorar el logro de los resultados esperados (Bas, 2011)(Moreno, 2005)(Ortega, 1990).
En el grupo GIRA, adicionalmente a la realización de múltiples actividades de capacitación y formación especialmente concebidas para estudiantes de semestres inferiores, tales como charlas, talleres, foros, conferencias, etc., se utiliza como ejercicio introductorio, hacer que los semilleros, asesorados por los demás actores intervinientes en su proceso de formación, lleven a cabo la construcción y puesta en funcionamiento de diferentes prototipos robóticos, desarrollados como actividades "hands-on" basados en aprendizaje activo (ver Figura 2).

\section{PLANTEAMIENTO DE LAS ACTIVIDADES}

Para los semilleros, los ambientes formativos que emplean herramientas didácticas, traen consigo numerosos aportes en conocimientos y habilidades que contribuyen al logro de mejores capacidades académicas e investigativas, fortaleciendo al mismo tiempo la motivación y el sentido de pertenencia al Grupo y al Programa Académico.

La estrategia consiste en plantear a los estudiantes el reto de hacer que un prototipo robótico cumpla una tarea específica, para lo cual pueden utilizarse los elementos y ayudas que estén a su alcance.

Esta actividad involucra la necesidad de ejecutar, tanto trabajos individuales como colaborativos, debido a la extensión y naturaleza de la misma, haciendo que cada participante pueda jugar diferentes roles, y sin que sea necesario contar con conocimientos ni habilidades previas. Sin embargo, si el grupo cuenta con experiencia en el tema, o si logran una rápida asimilación de los conceptos requeridos, esto permitirá que el tiempo de ejecución de cada reto resulte más corto y que se puedan plantear de nuevos desafíos.

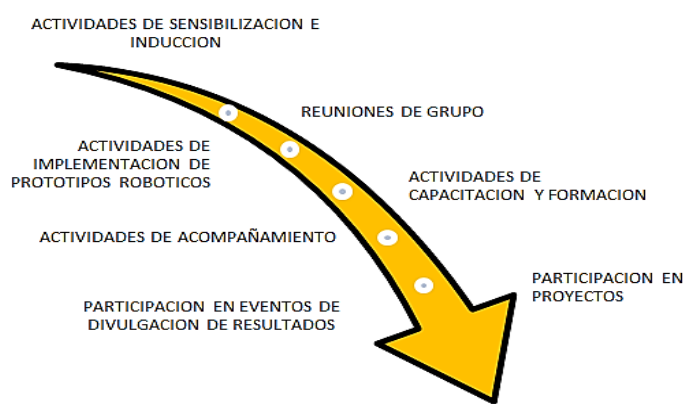

Fig. 2. Algunas actividades que hacen parte de la vinculación y formación de semilleros en el grupo GIRA. 
En estos ejercicios, se considera la robótica desde el enfoque de la robótica móvil y los manipuladores. Inicialmente, se opta por abordar la robótica móvil, ya que requiere menor complejidad para el ensamble, menos actuadores, tiene un modelamiento matemático más simple y requiere técnicas de control menos sofisticadas, entre otras.

Más adelante, se abordan actividades relacionadas con los manipuladores robóticos. Se emplean por lo general dos tipos herramientas de aprendizaje para la ejecución de este ejercicio:

- Hacer que los estudiantes construyan robots empleando técnicas "artesanales" en los que se usan elementos y materiales disponibles.

- Utilizar kits didácticos comerciales de robótica.

En estos ambientes, con el ánimo de simplificar la comprensión de conceptos, se divide al robot en cinco bloques funcionales principales: estructura mecánica, sistemas de actuación, sistemas de percepción, sistemas de suministro de energía y sistemas de control. Se hace hincapié en comprender que estos bloques interactúan entre sí para lograr que el prototipo robótico ejecute adecuadamente la tarea asignada.

Posteriormente se va profundizando en cada uno de los bloques funcionales para consolidar un conocimiento más elaborado y complejo que le permita al estudiante abordar retos más exigentes en el futuro. La Figura 4, ilustra las etapas en las que se divide la construcción de estos robots y sus componentes principales.

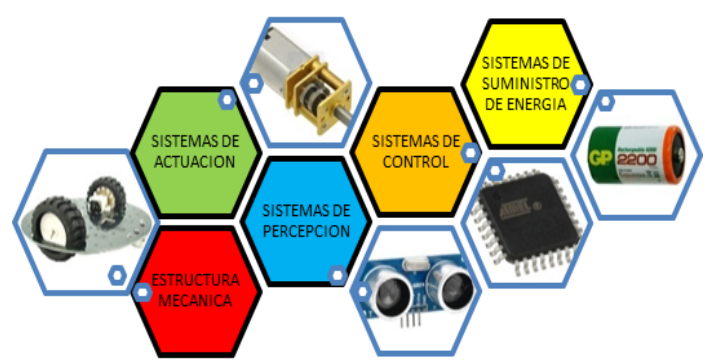

Fig.3. Bloques funcionales básicos para un robot móvil.

Fuentes: electro-project.blogspot.com, www.sigmaelectronica.net y www.gpbatteries.com

A continuación, se describen algunas experiencias llevadas a cabo en el grupo GIRA donde se utilizaron los dos tipos de herramientas de aprendizaje descritas anteriormente. Los ejercicios introductorios están basados en el montaje de diversas configuraciones y tareas a cumplir por parte del prototipo.

\section{EXPERIENCIA CON EL USO KITS DIDÁCTICOS}

Los kits didácticos de robótica ofrecen una buena opción para el de aprendizaje de la robótica y el desarrollo de habilidades de investigación, permitiendo optimizar el tiempo requerido para construir una plataforma, y facilitando la asimilación de conceptos y el uso de cada uno de sus componentes. Entre los kits más utilizados están LEGO Mindstorms, LEGO DACTA, Bioloid, Vex, Arduino, etc., los cuales se basan en la construcción de robots mediante el ensamble de piezas y la programación de acciones sencillas en ambientes de desarrollo altamente estructurados, a nivel de diagrama de bloques (Tello, 2013) (Velázquez, 2008)

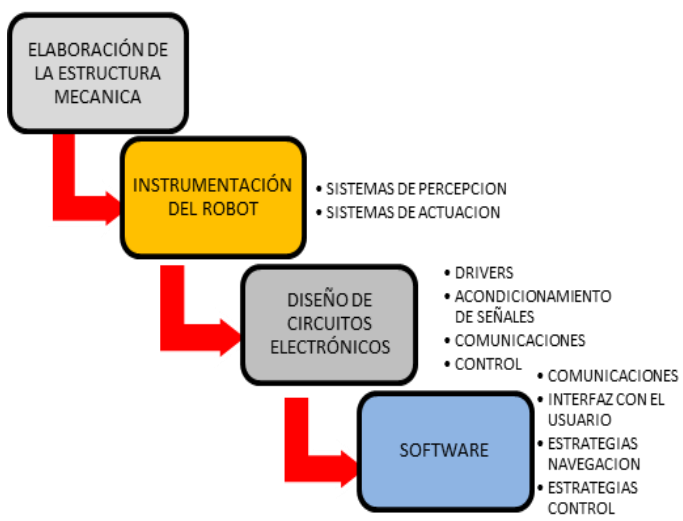

Fig.4. Esquema para la construcción de los robots en las actividades formativas.

El grupo GIRA posee diversas plataformas tales como LEGO DACTA, que incluyen gran cantidad de piezas para ensamblaje mecánico (bloques de construcción, engranajes, poleas, ejes, conectores, etc.), un bloque de control o "ladrillo" (brick) $\mathrm{RCX}$, motores y sensores para medir diversas variables.

Se cuenta también con kits LEGO MindStorms (versiones 1.0 y 2.0), kits Bioloid, plataformas para robótica móvil y manipuladores robóticos. Se dispone también de kits Arduino y tarjetas de desarrollo aplicables al control de robots. Los kits más utilizados en el grupo para el trabajo de formación de semilleros de primeros semestres son los kits LEGO. Las demás plataformas son abordadas posteriormente conforme se va adquiriendo más experiencia y conocimiento en el tema.

Los actores intervinientes en el proceso de formación del semillero, descritos en la Figura 1, 
participan en su capacitación, señalando las características de cada equipo, su forma de operación y los cuidados que se deben tener para evitar accidentes y preservar la integridad de los mismos.

Se inicia con un inventario de las piezas y luego, con ayuda del material de apoyo propio del grupo, los tutoriales incluidos en el software, las cartillas propias de cada kit y la información disponible en la web, se procede a construir los primeros modelos tomando usualmente como guía los ejemplos propuestos por el fabricante.

Considerando los bloques funcionales del robot, los componentes y elementos que hacen parte de estos kits se clasifican y emplean de la siguiente forma:

\subsection{Estructura mecánica}

Se pueden construir rápidamente diversas configuraciones de robots móviles utilizando las piezas estructurales y de conexión que se incluyen en cada kit.

Las piezas se clasifican en móviles, flexibles y de fijación. Los kits incluyen además una amplia gama de ruedas y orugas que permiten implementar la mayoría de las configuraciones para robots móviles, brindando estabilidad y/o velocidad según se requiera.

\subsection{Sistemas de actuación}

Se incluyen en cada kit hasta tres motores DC. Los motores incluidos en las primeras versiones LEGO DACTA (RCX) carecen de caja reductora y sensores de rotación; sin embargo, se dispone de un conjunto completo de engranajes y poleas que permiten crear los sistemas de reducción mecánica necesarios.

Los motores de la serie NXT en adelante, se caracterizan por tener una poderosa caja reductora y sensores de rotación incorporados, que permiten movimientos precisos y sincronización con otros motores.

\subsection{Sistemas de percepción}

Las primeras versiones contaban con algunos tipos de sensores y dispositivos, que son versiones primitivas de los componentes actuales (temperatura, luz, tacto, rotación, etc.). Los sensores básicos de las versiones NXT son: luz, sonido, tacto, color, ultrasonido y rotación (integrado en los motores).
Los sensores de color, luz, sonido y tacto son dispositivos de entrada análogos, y el sensor ultrasónico se clasifica como dispositivo de entrada digital (Pozo, 2000), de modo que tiene una amplia gama de posibilidades de detección de señales y el manejo de los conceptos relacionados (Griffin, 2010) (NI, 2009).

Para el NXT, se dispone comercialmente de otros sensores que incrementan las prestaciones del equipo como son el compás magnético, sensores de ángulo, sensores de fuerza, etc. (Hitechnic, 2014).

La amplia gama de posibilidades permite que los semilleros puedan trabajar con la edometría y la navegación del móvil, necesarias para la realización de proyectos que van desde básicos hasta avanzados.
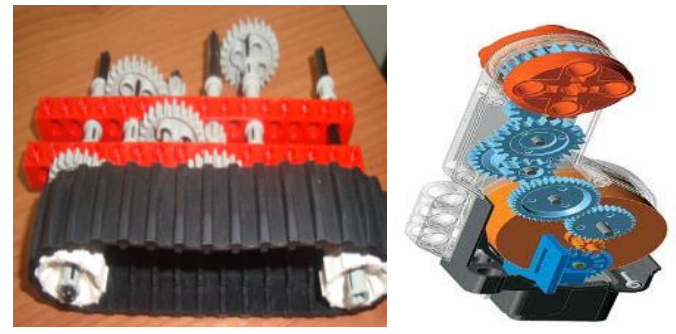

Fig. 5. Caja reductora y oruga (kit Lego Dacta) y, esquema interno del servomotor NXT.

Fuente: http://raor.ru/equipment/catalog/catalog_52.html

\subsection{Sistemas de suministro de energía}

La primera versión de la unidad de control o ladrillo del LEGO (RCX) podía ser alimentado tanto por una fuente externa como por un grupo de seis baterías AA. Los modelos más recientes solo permiten alimentación por baterías, ofreciéndose (solo para el caso educacional), un módulo de alimentación que cuenta con una batería recargable. En este punto se hace énfasis en que la autonomía de la batería es función de la cantidad de equipamiento conectado y de las rutinas de operación y/o control implementadas.

\subsection{Sistemas de control}

Hardware: Las unidades de control de los kits (LEGO RCX, el NXT y el nuevo EV3) contienen una mini-computadora o ladrillo compacta, compuesta por un microprocesador y/o algunos microcontroladores, que incluyen puertos de entrada (que se usan para conectar los sensores disponibles) y puertos de salida usados para conectar los motores. Un aspecto positivo, y a la vez negativo para el aprendizaje, es que los drivers de los motores y los sistemas de 
acondicionamiento, ya están incluidos tanto en cada dispositivo como en la unidad de control, por lo que estos conceptos son transparentes al usuario. Software: Las unidades de control se pueden programar desde una computadora (utilizando el software ROBOLAB, LEGO-G o versiones más modernas), que se caracterizan por ser entornos muy amigables con el usuario y altamente intuitivos, permitiendo realizar desde programas muy básicos hasta algunos muy complejos sin necesidad de ser un experto en programación. Otro aspecto que los hace propicios para el trabajo con semilleros, y en particular con semilleros de ingeniería electrónica, es que son entornos compatibles con el software LabVIEW de National Instruments y otros software de programación tales como Robotics Developer Studio, Not eXactly C, LeJOS, RobotC, y PyNXC.

Una vez conocidos los bloques que conforman cada kit, los semilleros proceden a armar libremente diferentes configuraciones, limitados solamente por la creatividad y el número de piezas y componentes incluidos. Los kits ofrecen la ventaja de requerir tiempos promedios muy cortos para el armado de cada estructura (en el orden de minutos a horas).

Por otro lado, existen, tanto en el software como en la web, gran cantidad de ayudas que facilitan materializar las ideas y proyectos. En unas pocas horas, por ejemplo, un estudiante sin experiencia puede llegar a construir y programar, su propio robot móvil seguidor de línea. La Figura 6 muestra algunos de los robots armados por los semilleros de investigación en estas actividades introductorias.
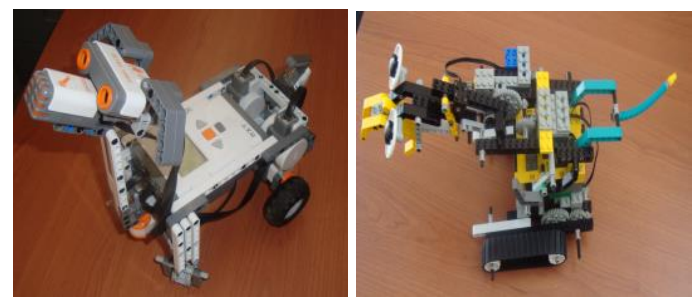

Fig. 6. Robot zoomórfico en configuración diferencial (kit LEGO NXT) y robot zoomórfico orugado armado (kit LEGO DACTA)

\section{EXPERIENCIA CON EL USO DE ROBOTS ARTESANALES}

Los proyectos que realizan los semilleros de investigación en los que la creación y construcción de los prototipos involucran técnicas "artesanales", son aquellos donde los bloques fundamentales que componen en robot son "creaciones propias" de los mismos semilleros. El procedimiento descrito a continuación se basa en una generalización de las actividades que se desarrollan frecuentemente en el grupo GIRA, sin embargo, en algunas ocasiones pueden existir variaciones.

En la experiencia con robots artesanales se empieza por establecer la tarea a cumplir por parte del robot, en la que el reto se traslada a la construcción y puesta en funcionamiento de cada uno de los bloques necesarios. Se deben identificar los componentes y materiales necesarios, estableciendo su disponibilidad (inventario del grupo o comercio local), así como las limitaciones de presupuesto (Torres et al., 2013).

La Tabla 1 ilustra algunos de los elementos y materiales con los que se ejecutan comúnmente estas actividades.

Tabla 1: Materiales y elementos disponibles comúnmente para la construcción de prototipos.

\begin{tabular}{|l|l|}
\hline BLOQUE & \multicolumn{1}{|c|}{$\begin{array}{c}\text { MATERIALES O ELEMENTOS } \\
\text { DISPONIBLES }\end{array}$} \\
\hline $\begin{array}{l}\text { Estructura } \\
\text { mecánica }\end{array}$ & $\begin{array}{l}\text { MDF, fibra de vidrio, cartón paja, polímeros } \\
\text { (Poliestireno, acrílico, etc.) y láminas } \\
\text { metálicas (Aluminio y Acero Galvanizado) } \\
\text { Ruedas, partes de juguetes, tuercas, tornillos } \\
\text { y pegamentos }\end{array}$ \\
\hline $\begin{array}{l}\text { Sistemas de } \\
\text { actuación }\end{array}$ & $\begin{array}{l}\text { Motores DC, moto reductores DC y } \\
\text { servomotores } \\
\text { Drivers de potencia. }\end{array}$ \\
\hline $\begin{array}{l}\text { Sistemas de } \\
\text { percepción }\end{array}$ & $\begin{array}{l}\text { Sensores ópticos infra rojos CNY70 y } \\
\text { QRD1114, fotorresistencias, LED y sensores } \\
\text { infra rojos }\end{array}$ \\
\hline $\begin{array}{l}\text { Suministro } \\
\text { de energía }\end{array}$ & $\begin{array}{l}\text { Baterías, fuentes externas de alimentación } \\
\text { (reguladas) y baterías recargables (NiMH e } \\
\text { Ion de Litio). }\end{array}$ \\
\hline $\begin{array}{l}\text { Sistemas de } \\
\text { control }\end{array}$ & $\begin{array}{l}\text { Circuitos con semiconductores básicos, } \\
\text { y Freescale), dispositivos } \\
\text { programables (FPGAs o CPLDs), SoCs y } \\
\text { computadoras. }\end{array}$ \\
\hline
\end{tabular}

Una vez identificada la información de partida, los semilleros, con el apoyo de los actores intervinientes, proceden a realizar el diseño e implementación de cada uno de los bloques constitutivos del robot. Las actividades llevadas a cabo para cada bloque se describen a continuación.

\subsection{Estructura mecánica}

Para esta etapa se realizan charlas orientadas por el joven investigador, o algún docente del grupo, para analizar las características de las estructuras mecánicas, especialmente las relacionadas con los 
métodos de locomoción. Cada semillero por su cuenta debe complementar esta información consultando bibliografía, artículos científicos, videos, etc. Se abordan las configuraciones Ackerman, triciclo, diferencial, skid steer, orugadas, síncronas y sistemas de locomoción basados en patas. Por practicidad se inicia comúnmente con la configuración diferencial, aunque se han usado también configuraciones triciclo y Ackerman (especialmente cuando se requiere velocidad).

La selección del material para la construcción del prototipo puede ser variada y obedece a la disponibilidad de tiempo y recursos económicos. El uso de aluminio, acero galvanizado o poliestireno puede producir buenos resultados, pero a su vez puede incrementar los costos y tiempos de desarrollo, además de requerir que se posean o desarrollen, habilidades especiales para manejo de estos materiales. El MDF es un material que puede dar buenos resultados y es fácil de utilizar requiriendo solo el uso de herramientas elementales.

\subsection{Sistemas de actuación}

La selección de los motores se debe realizar cuidadosamente ya que puede afectar el funcionamiento del sistema $\mathrm{y}$ el presupuesto requerido. Los moto-reductores se encuentran entre los más utilizados por su versatilidad y buenas características de torque. Frecuentemente, dentro de los sistemas de actuación se incluye también el diseño de los circuitos de potencia o drivers, dedicados al manejo de las corrientes y voltajes demandados por cada dispositivo. Los circuitos integrados de potencia, como los puentes L293 y L298, se utilizan a menudo por su simplicidad y bajo costo.

\subsection{Sistemas de percepción.}

Los sistemas de percepción para el desarrollo de aplicaciones robóticas son básicamente de dos tipos: los requeridos para percibir información propia del equipo (sensores propioceptivos) y los necesarios para tomar información del entorno donde el robot se desempeña (exteroceptivos). Dentro de los sensores más utilizados están los sensores de contacto (finales de carrera, pulsadores, etc.), los ópticos de proximidad o de detección de color (LDRs, QRD1114, el CNY70 y los infrarrojos SHARP GP2Y, entre otros), los ultrasónicos, los encoders y sensores de efecto Hall.
En esta etapa se realizan actividades orientadas a que los semilleros reconozcan, mediante la experiencia, que algunos sensores pueden resultar más susceptibles que otros a las condiciones del ambiente y/o de operatividad, lo que puede afectar considerablemente el desempeño del robot.

\subsection{Sistemas de suministro de energía}

Para el suministro de energía se puede emplear una fuente de alimentación externa al robot o recurrir al uso de baterías (generalmente recargables del tipo Ion Litio o NIMH). El uso de una fuente externa tiene la desventaja de requerir el empleo de un cable umbilical, lo que limita drásticamente la libertad de movimientos y afecta la dinámica del equipo. Las baterías por su parte tienen una limitada autonomía y pueden afectar el peso y centro de masa del robot.

Adicionalmente, se debe tener en cuenta que el voltaje de las baterías desciende durante su operación y que usualmente se requiere emplear un regulador de voltaje para asegurar la correcta operación de los circuitos alimentados por estas. En algunos casos es necesario implementar también circuitos básicos de carga de baterías como parte del sistema de suministro de energía.

\subsection{Sistemas de control.}

Este es tal vez uno de los bloques más importantes durante el diseño e implementación del robot. Pueden explorarse diversas alternativas que van desde circuitos lógicos de tecnología LSI/MSI (circuitos combinacionales o secuenciales básicos), dispositivos de lógica reconfigurable (FPGAs, CPLDs), microcontroladores, microprocesadores, SoCs o DSPs.

Generalmente, se opta primero por utilizar los circuitos y tecnologías más simples, para los que se analizan sus capacidades y limitaciones. Posteriormente los semilleros reciben capacitación para aprender a utilizar las tecnologías más avanzadas y flexibles.

Una vez finalizado el proceso de desarrollo de todos los bloques, el semillero realiza la integración de los mismos y pone a prueba su montaje para verificar si éste cumple adecuadamente con la tarea asignada. A pesar de que se contempla que en cada etapa se realicen pruebas de funcionamiento básicas de cada bloque, es común que en conjunto no se obtengan los resultados esperados. Muchas veces la tarea de encontrar los inconvenientes y de plantear y 
ejecutar soluciones, resulta más enriquecedora que la misma actividad de implementación.

La Figura 7 muestra un robot seguidor de línea de configuración diferencial, que fue elaborado por estudiantes vinculados al semillero de investigación del grupo GIRA, que se encontraban entre el primero y tercer semestre de Ingeniería Electrónica.

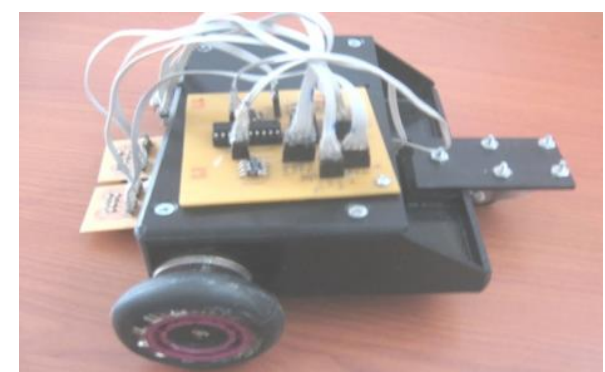

Fig. 7. Plataforma robótica desarrollada artesanalmente por estudiantes semillero.

El desarrollo de este robot involucró la construcción de la estructura en poliestireno, la adecuación de los sistemas de locomoción (motoreductores), la disposición de los sensores CNY70, la elaboración de tarjetas electrónicas para la conexión de los sensores y el dispositivo de control basado en un microcontrolador PIC 16F84A de Microchip.

El tiempo de ejecución de este proyecto fue de cuatro meses, con sesiones de dos horas semanales. Durante el desarrollo de este proyecto se contó con el acompañamiento de docentes, el joven investigador adscrito al grupo y algunos semilleros de investigación de últimos semestres.

\section{RESULTADOS DE LAS ACTIVIDADES DESARROLLADAS}

Las dos herramientas didácticas utilizadas en este enfoque, constituidas por el uso de kits robóticos comerciales y de robots implementados artesanalmente, desarrolladas como actividades formativas al interior del semillero, han permitido obtener resultados interesantes en los procesos de vinculación y formación de estudiantes de primeros semestres al semillero del grupo GIRA.

El uso de los dos tipos de robots propuestos y las actividades que se derivan de su implementación, permiten atacar objetivos de aprendizaje diferentes para los estudiantes que quieren adentrarse en el mundo de la robótica.
La Tabla 2 señala algunas fortalezas y debilidades que se han identificado durante el uso de estas dos herramientas.

Tabla 2. Algunas fortalezas y debilidades identificadas para las herramientas utilizadas

\begin{tabular}{|c|c|}
\hline \multicolumn{2}{|c|}{ USO DE KITS DE ROBÓTICA } \\
\hline Fortaleza & Debilidad \\
\hline $\begin{array}{l}\text { Tiempos cortos de } \\
\text { implementación. } \\
\text { Reconfigurabilidad } \\
\text { (hardware y software) } \\
\text { Abundante información de } \\
\text { soporte disponible. } \\
\text { Resistencia, robustez y } \\
\text { seguridad. } \\
\text { Software sencillo, } \\
\text { amigable e intuitivo, con } \\
\text { capacidad de ser enlazable } \\
\text { con otros de mayor nivel y } \\
\text { complejidad. }\end{array}$ & $\begin{array}{l}\text { Costo relativamente elevado. } \\
\text { Limitaciones por cantidad y/o } \\
\text { tipo de piezas disponibles. } \\
\text { Características mecánicas, } \\
\text { eléctricas y de software } \\
\text { condicionadas por las piezas y } \\
\text { demás componentes. } \\
\text { Baja compatibilidad con } \\
\text { equipos y dispositivos de } \\
\text { otros fabricantes. }\end{array}$ \\
\hline \multicolumn{2}{|c|}{$\begin{array}{l}\text { USO DE ROBOTS IMPLEMENTADOS CON } \\
\text { TÉCNICAS ARTESANALES }\end{array}$} \\
\hline 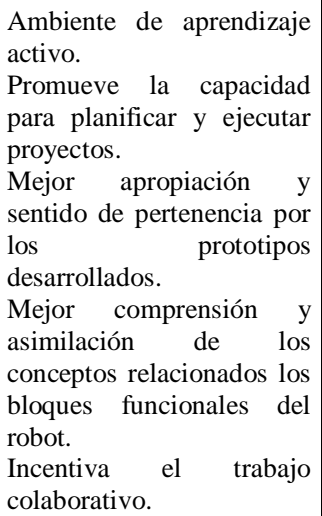 & 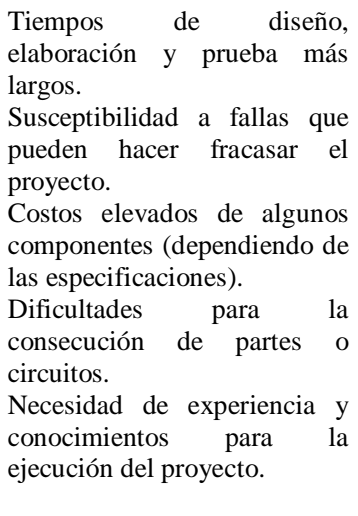 \\
\hline
\end{tabular}

Las habilidades y conocimientos adquiridos por cada estudiante, fruto de su interacción con estos ambientes de aprendizaje, han contribuido a mejorar su desempeño académico e investigativo a lo largo de su permanencia en la universidad.

Dentro de las habilidades generales que pueden desarrollarse $y / o$ fortalecerse con estas actividades están por ejemplo las relacionadas con la creatividad, la capacidad para solucionar problemas, el trabajo colaborativo, el uso de herramientas tecnológicas, la capacidad de autoaprendizaje, la capacidad de socializar resultados de investigación, entre otras.

Los buenos resultados obtenidos han propiciado a que permanentemente se cuente con un número significativo de estudiantes (más de 20) en el semillero de investigación, los cuales apoyan los diferentes proyectos que se desarrollan en el grupo, 
especialmente los relacionados con robótica educativa. Adicionalmente, los semilleros motivados por su trabajo en el grupo y por las experiencias adquiridas en su fase de formación en el mismo, registran una alta participación en eventos especializados, como los encuentros regionales, nacionales e internacionales de Semilleros de Investigación - Red Colsi.

\section{CONCLUSIONES}

Se observa que los estudiantes que han hecho parte de este proceso, y que por largo tiempo han permanecido vinculados con el grupo de investigación, muestran mayores niveles de participación en eventos de carácter científico e investigativo (con ponencias nacionales e internacionales) que los estudiantes que no lo han hecho. Adicionalmente, estos estudiantes acceden con mayor frecuencia a los estímulos institucionales por investigación (tales como becas de investigación y convocatorias de jóvenes investigadores), y muestran mejores niveles de rendimiento académico.

Los ambientes de aprendizaje desarrollados permiten una adecuada interacción entre los actores que intervienen en los procesos formativos en el grupo de investigación, permitiendo beneficiar especialmente la dinámica académica e investigativa de los estudiantes vinculados al semillero.

El uso de las dos herramientas didácticas planteadas, (kits robóticos y robots diseñados e implementados con técnicas artesanales), ofrece una perspectiva más completa que cuando se abordan estas dos alternativas por separado, ya que se exploran las potencialidades e inconvenientes de cada una, y se plantean soluciones que enriquece el proceso de aprendizaje.

\section{REFERENCIAS}

Almenara. J.C. (2009) Las TIC y el desarrollo de las competencias básicas. Una propuesta para la educación primaria. Editorial ESIC. Madrid

Ausubel, D. P. (2002). Adquisición y Retención del Conocimiento, Una perspectiva cognitiva. Editorial Paidós Mexicana. México.
Barrera L., N. \& Mesa M. L. A. La robótica educativa como instrumento didáctico alternativo en educación básica. Revista Colombiana de Tecnologías de AvanzadaUniversidad de Pamplona, Volumen 2-Numero 22-Año 2013. ISSN 1692-7257.

Bas. P. E. Aprendizaje basado en problemas. Cuadernos de pedagogía, no 409 (Febrero 2011), 42 - 44p.: bib.

Griffin, T. The Art of LEGO Mindstorms NXT-G Programming. (2010). San Francisco, CA. No Starch Press.

Moreno C. M. (2005). Desarrollo de ambientes de aprendizaje en educación a distancia. Textos de encuentro de educación a distancia. Universidad de Guadalajara. México.

National Instruments [NI]. LabVIEW- Lego Mindstorms NXT Module Programming Guide. (2009). Austin, TX: NI.

Torres Clayton José, Archila John Faber, Tronco Mário Luiz, Becker Marcelo, Viera Porto Arthur José, Tiberti Alexander José. (2013). Estudio cinemático de una plataforma robótica para agricultura. Revista colombiana de tecnologías de Avanzada. 2 (22). Pág. 131 137.

Ortega R, R. (1990). Jugar y aprender: una estrategia de intervención educativa. Editorial Diada, 1990. Sevilla España.

Papert, S. (1995). La Máquina de los niños: Replantearse la educación en la era de los ordenadores. Editorial Paidos. Barcelona.

Pozo J. I. (2000) Aprender y enseñar ciencia. Segunda edición. Editorial Morata. España.

Restrepo G., Bernardo. Conceptos y Aplicaciones de la Investigación Formativa, y Criterios para Evaluar la Investigación científica en sentido estricto. Consejo Nacional de Acreditación CNA, Colombia. 2001

Ruiz V. S. (2007) Educa trónica: Innovación en el aprendizaje de las ciencias y la tecnología. Editorial Díaz Santos. México.

Tello. L E. et al. Revisión de la plataforma robótica LEGO Mindstorms para aplicaciones educativas y de investigación. Revista S\&T, 11(26), 9-27

Velásquez N. J. (2008). Ambientes Lúdicos de Aprendizaje Diseño y operación. Editorial Trillas. México. 